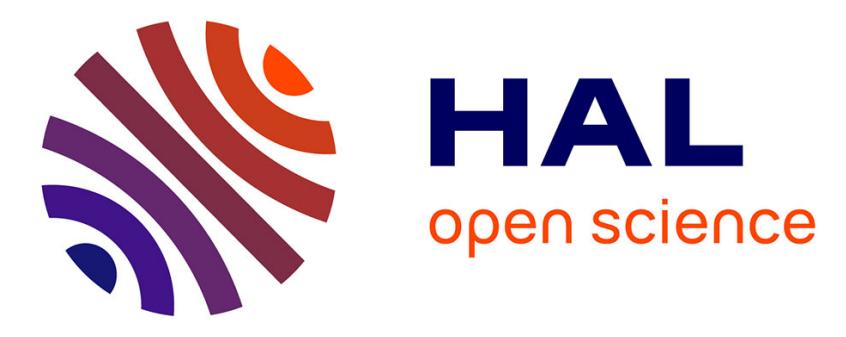

\title{
Wind Networking Applied to Aircraft Trajectory Prediction
}

Karim Legrand, Christophe Rabut, Daniel Delahaye

\section{To cite this version:}

Karim Legrand, Christophe Rabut, Daniel Delahaye. Wind Networking Applied to Aircraft Trajectory Prediction. DASC 2015 IEEE/AIAA 34th Digital Avionics Systems Conference, Sep 2015, Prague, Czech Republic. pp.1A4-1-1A410 / 978-1-4799-8939-3, 10.1109/DASC.2015.7311330 . hal-01224188

\section{HAL Id: hal-01224188 \\ https://hal-enac.archives-ouvertes.fr/hal-01224188}

Submitted on 5 Nov 2015

HAL is a multi-disciplinary open access archive for the deposit and dissemination of scientific research documents, whether they are published or not. The documents may come from teaching and research institutions in France or abroad, or from public or private research centers.
L'archive ouverte pluridisciplinaire HAL, est destinée au dépôt et à la diffusion de documents scientifiques de niveau recherche, publiés ou non, émanant des établissements d'enseignement et de recherche français ou étrangers, des laboratoires publics ou privés. 


\title{
WIND NETWORKING APPLIED TO AIRCRAFT TRAJECTORY PREDICTION
}

\author{
Karim LEGRAND, ENAC, 7 Avenue Edouard Belin, 31055 Toulouse, France \\ Christophe RABUT, INSA, 135 Avenue de Rangueil, 31400 Toulouse, France \\ Daniel DELAHAYE, ENAC, 7 Avenue Edouard Belin, 31055 Toulouse, France
}

\section{Abstract}

Trajectory prediction estimates the future position of aircraft along their planned trajectories in order to detect potential conflicts and to optimize air space occupancy. This prediction is a critical task in the Air Traffic Control (ATC) process and has been studied for many years. For the future automation processes developed in the SESAR [1], NextGen [2] and CARATS [3] projects, such trajectory prediction will be even more critical. In these projects the trajectory predictors generate aircraft forecast trajectories, typically for client applications. As there is always a deviation between the predicted wind (from the weather forecasts) and the encountered wind, the main longitudinal (along-track) error source between the predicted and the actual trajectory is linked to wind estimation. Based on the current performances of Air Traffic Control systems, controllers are able to efficiently detect conflict 20 minutes in advance ; for a larger time horizon (look-ahead time), the induced trajectory prediction uncertainty strongly reduces the reliability of the conflict detection. The goal of this work is to measure the potential benefit produced by sharing wind measures between aircraft (this concept will be called wind networking $(W N))$. To reach this goal, aircraft measure their local atmospheric data (wind, temperature, density and pressure) and broadcast them to the other aircraft. Having such distributed weather information, each aircraft is able to compute an enhanced local wind map as a function of location (3D) and time. These updated wind fields could be shared with other aircraft and/or with ground systems. Using this enhanced weather information, each aircraft is able to improve drastically its own trajectory prediction. This concept has been simulated in the French airspace with 8000 flights. Comparisons have been investigated on trajectory prediction performances with and without wind networking. Statistics have been conducted in order to measure the benefit of such concept in both time and space dimensions showing higher improvement in high traffic areas, as expected.

\section{Introduction}

The current Air Traffic Management (ATM) system is based on a sectorized airspace and predetermined routes. Routes and sectors are operated according to the air traffic flow through AirSpace Management (ASM). When the air traffic volume exceeds the air traffic control capacity, air traffic controllers instruct ground delays (i.e slots), air delays (speed reductions, holds, ...) or alternative routes. Current improvements come from the design and the implementation of automated flight paths that rely on Performance Based Navigation (PBN) to facilitate airspace design, traffic flow management and runways utilization. Air Traffic Management is composed of a number of complementary systems :

- Airspace management (ASM)

- Air traffic flow and capacity management (ATFCM)

- Air traffic control (ATC).

These systems together, make sure that flights are safe and on schedule.

Initiatives, based on 1998 ICAO ${ }^{1}$ Global ATM Operational Concept [4], have been taken to improve the safety and efficiency of air transportation through major projects like NextGen [2] in the USA, SESAR [1] in Europe and CARATS [3] in Japan. All these projects need to optimize the arrivals to airports through the emerging Trajectory Based Operations $(T B O)$ concept. The TBO is based on knowing and sharing the current and planned aircraft positions. This means that aircraft are constrained in a spatio-temporal space, i.e a 4 Dimensions (4D) space $(3 \mathrm{D}+\mathrm{T})$.

\footnotetext{
${ }^{1}$ International Civil Aviation Organization
} 
Some of the expected benefits are [5] : traffic synchronization, organized flow of traffic, flexible capacity management, adjustments in airspace capacity to variations in demand and delegation of separation to flight deck.

NextGen, SESAR and CARATS rely on the 4D trajectory concept. By introducing a fourth parameter in the trajectory, time constraints on specific waypoints may be negotiated between the flight crew and the air traffic controllers in order to sequence the traffic and to reduce congestion in sectors. This new concept introduces time-based management in all phases of flight.

To address the flexibility requested by air carriers, these projects assume that a 4D trajectory is negotiated via a datalink between the ATC and the aircraft before push-back and up to the arrival gate. The data are exchanged directly between the Flight Management System (FMS) and ground systems.

The flip side of the coin is that more precise information is required on the aircraft position at any given moment, i.e current position and predicted position, or in other words the look-ahead time must be increased. As explained in [6] errors in wind estimation lead to ground speed errors and cumulative along-track error between $-8 \mathrm{NM}$ and $+8 \mathrm{NM}$ when the wind has not been updated during the last 30 minutes. Practically for a jet flying at $0.8 \mathrm{M}$ it means 1 minute ahead or after schedule over the next half hour expected position.

Trajectory prediction capabilities are an essential part for most, if not all, Air Traffic Management Decision Support Tools (DST). Most of the DST are provided with their own unique trajectory prediction capability and the main objective of our work is to develop a common trajectory prediction algorithm.

Controllers monitor the air traffic situation by surveillance system. This system is critical for all ATC operations other than at control towers in good visibility, when the controllers can directly observe the air traffic. A key concept of future ATM systems is Required Monitoring Performance (RMP), which is intended to specify an aircraft trajectory prediction capability and its related accuracy, integrity and availability of a monitoring system for a given sector of airspace and/or phase of operation. Surveillance grants both aircraft tactical separation, and strategic planning of traffic flows. The primary objective of the surveillance function is to support the following types of airspace management functions :

- Short Term Separation Assurance

- Medium Term Separation Assurance

- Medium Term Airspace Planning

- Strategic/Long Term Planning and Flow Management

Future flow management system goals to transition from a departure managed system to an arrival managed system of flow management. An accurate 4D trajectory prediction from departure to arrival enables a technology for strategic management by providing accurate state and intent information for long term path predictions.

\section{Trajectory Prediction Problem}

When a controller observes traffic on the radar screen, he tries to identify convergent aircraft that may be in conflict in the near future, in order to apply maneuvers that will keep them separated. The problem is then to estimate the next aircraft positions within a 10 to 30 minutes time horizon. A 4-dimensional (4D) trajectory prediction contains data specifying the predicted horizontal and vertical positions of an aircraft over a given time period. The ability to accurately predict trajectories for different types of aircraft under different flight conditions, which include external actions (pilot, ATC) and atmospheric influences (wind, temperature), is an important factor when determining the accuracy and effectiveness of an ATM system.

A major concern when dealing with trajectory prediction is the ability to assess a goodness-offit value to the forecast trajectory compared with the original one. Many different factors may distort the prediction, their weights depend on the forecast time horizon. Theoretically, the knowledge of the flight dynamics equations for a given aircraft, the intended flight plan and exogenous parameters like temperature, wind and ATC controllers instructions should be enough to accurately model a trajectory from departure to destination. Unfortunately, many of these factors are unknown or partially known. A classical way of modeling such uncertainties is to assume that they are realizations of some random process (known from statistical estimators that can be computed using measured data). This induces a residual noise of trajectory prediction that comes after 
a time integration with a growing covariance matrix indicating that the estimated position is less and less accurate. The current limit is around 15 minutes if one wants to keep trajectory prediction usable, specially for early conflicts detection.

The problem of aircraft trajectory prediction involves many uncertain factors such as wind, pressure, aircraft weight, etc... Their influence strongly affects the quality of prediction when time horizon increases. Let us briefly describe some of them.

- Weight. Aircraft weight mainly depends on number of passengers, luggage, freight and fuel on board.

- Pilot Actions. Such actions are taken to follow the flight plan, to avoid adverse weather conditions or when controllers change the flight path for conflict resolution purpose.

- Wind. Wind is the major factor impacting trajectory prediction. Furthermore, wind uncertainty is spread in time and in space.

- Temperature. Air temperature is linked to air density ( $\rho)$ which drives aircraft drag $d=\frac{1}{2} c_{x} \rho S V^{2}$ where $S$ is the wing surface, $\mathrm{V}$ is the aircraft air speed and $c_{x}$ is a coefficient. It is also linked to the thrust limit of the engines. Maintaining a given Mach under increased temperature conditions equals increasing true air speed, and in warm temperatures thrust limit may prevent the crew from maintaining the flight plan mach number. As for the wind, temperature error is spread in time and space.

- Aircraft Trajectory Model. Several aircraft trajectory models can be applied for trajectory prediction with more or less accuracy. The more information about aircraft is available, the best the prediction will be produced by such a model. Any model induces a modeling error which has to be minimized in order to improve the trajectory prediction. In this sense, the aircraft model choice is also a limiting factor. All aircraft models, including tabular ones, are based on solving ordinary differential equations. The control input includes initial condition and model parameters. Refinement (and computational complexity) ranges from tabular to many degrees of freedom. There is always a trade-off between accuracy and smoothness.

- Measurement errors. The main measurement er-

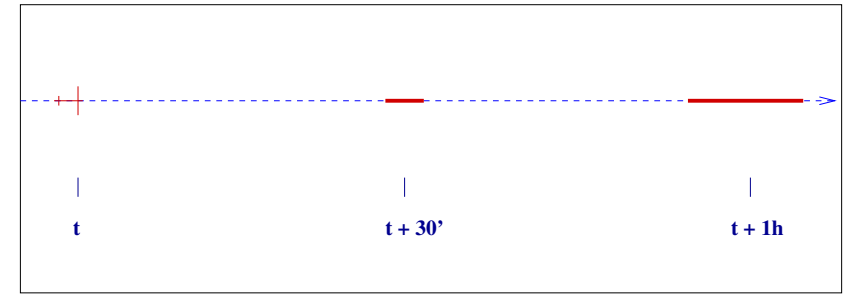

Figure 1. Trajectory prediction limitations

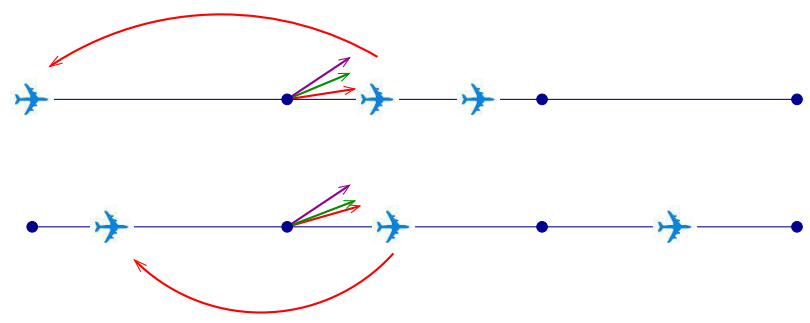

\section{Figure 2. Oceanic Wind Networking Concept}

ror is due to the radar trackers used to estimate the aircraft current position.

Due to the stochastic nature of such perturbation factors, trajectory prediction becomes inefficient after a given period of time (about 15 minutes for conflict detection purpose). Figure 1 illustrates the trajectory prediction error evolving with time. On this figure, $t$ is the current position timestamp, $t+10$ and $t+20$ denote the future prediction horizon, dark areas show the possible future aircraft positions.

Several efforts have been made to improve the trajectory prediction by better wind estimation [7][11].

Our work tried to improve Trajectory Prediction (TP) accuracy, not by estimating the wind errors but by continuously updating the wind data available on board using the wind data available from the neighboring aircraft. The wind data refresh cycle could be reduced to less than 15 minutes using this concept. This concept has already been studied for oceanic airspace and has produced very good results [12]. In this case, each aircraft back propagates its measured wind to the next following aircraft on the same oceanic track as shown on the Figure 2. The benefit associated to such wind sharing concept reduces the time error at reporting position from few minutes to few seconds.

In the present work we propose to study the 
benefits of such a concept for tactical application mainly to improve the near term trajectory prediction.

The first part of the paper describes the wind networking concept and how it could be applied to aircraft trajectory prediction. The second part presents the algorithm used to implement the $\mathrm{WN}$ and proposes smooth vector interpolation approach. The third part introduces the framework used for our simulations and demonstrates the benefit of WN of trajectory prediction for a large airspace (France airspace).

\section{Concept description}

The Wind Networking concept is based on modern aircraft capacity to measure atmospheric data through their Air Data Computer ADC. Plenty of accurate (i.e not derived from a numerical weather model) temperature wind data are available in every controlled airspace. We assume that in a near future aircraft will be able to exchange such information through aircraft to aircraft data link, or aircraft to ground data link [13].

During every controlled flight, an aircraft crosses control sectors and aircraft trajectories. If by any mean past data derived from its ADC is stored on board, it can be transferred to :

- other aircraft planning to fly a trajectory in the vicinity of the already flown trajectory,

- or to Air Traffic Control Center in charge of the already crossed airspace.

In order to illustrate the Wind Networking concept we will consider the B737 practical case. Most crews use a technical flight plan prepared by the company operations to fill the Flight Management System (FMS) route. Taking the example of Smith Industries B737 FMS, the crew is supposed to fill the wind for the chosen cruising level (CRZ WIND) field in the FMS which linearly interpolates the climb wind from zero to the top of climb wind value, and propagates it to the route legs if the route has already been entered.

To verify the fuel balance and the Estimated Times of Arrival (ETA)s before take-off the crew is supposed to enter (or uplink) the predicted winds in the FMS. On very short flights most of the time there is little reason to enter several en route winds. On long range flights omitting forecast winds, or filling the FMS with erroneous winds, may lead up to erroneous fuel consumption predictions ending with a diverting

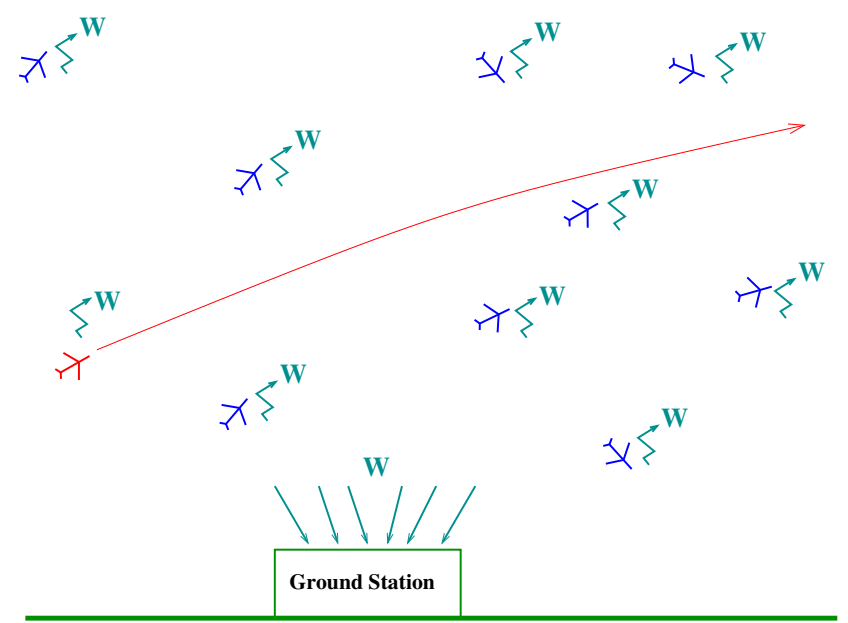

Figure 3. Wind Networking Principle

flight. Obviously, as soon as airborne, accurate wind values are needed to give most accurate ETAs and fuel predictions.

Our concept is simple, each time a more recent wind is available, it has to be "uplinked" to the FMS. This update is not limited to one flight level (e.g the currently or planned flight level), but provides an update of the predicted winds actually encountered by previous flying aircraft. Some advantages are better after take-off fuel consumption estimations (i.e better chances for a true optimal flight level), better trajectory prediction (e.g accurate ETA), better Top $O f$ Descent (TOD) estimation for idle thrust descents [14] and Continuous Descent Approach CDA [15], [16] which also means less noise on overflown cities during the descend and approaches phases [17].

The concept may be summarized in both (see Figure 3) :

- near real time aircraft/aircraft wind information sharing,

- near real time aircraft/ground wind information sharing.

\section{Algorithm}

The algorithm we have been developing to demonstrate the benefit of tactical wind networking concept is based on wind prediction improvement by using wind measures from other aircraft in the 4D vicinity of a given aircraft.

First we consider a large set of aircraft in order to have relevant statistical results. In our case, we will consider the traffic over a European country. 
For each trajectory sample, one must be able to locate the neighboring aircraft in a 4 dimensional space. The naive approach consists in a pairwise comparison which is dramatically inefficient. For instance, if we consider 8000 trajectories over the French airspace with an average observation time of two hours, sampled every 10 seconds (radar period), we get $8000 \times 2 \times 360=5760000$ samples. This means that if we want to find the neighboring aircraft for a given sample, we have to compute 5760000 distances, and identify the closest ones. Furthermore, this computation has to be done for every trajectory sample, meaning that the total distance computation is $5760000 \times 576000=3,3 \times 10^{12}$. If one distance computation costs $10^{-9}$ second, the duration of the whole distances computation lasts $\simeq 0.9$ hour, which is too much.

In order to avoid this brute force computation, a 4D grid has been built in which each trajectory sample has been inserted. Each point of the 8000 trajectory is thus identified by four grid coordinates. An example of such a grid is given on Figure 4 where we are searching current trajectory (red dot). As shown on the figure, only the samples located in the red square will be checked in terms of distance to the red dot. A 2D grid has been used for representation, but the real grid has been built in 4D. Each sample (red dot in the figure) has coordinates, $(5,6)$ in the example and only the neighboring boxes have to be checked. In order to validate this approach, one must select the boxes dimensions.

In a first step, wind maps are inserted in the 4D grid as shown on Figure 5 where the predicted wind and the true wind are stored on each grid point.

Then each trajectory is inserted in the grid and the computation of the trajectory prediction improvement is done into two steps. The first step updates, when possible, the wind on trajectories, meaning having some aircraft which has already measured some wind in the current aircraft 4D neighborhood (in space and in time). For our application, neighborhood means areas where the wind does not change too much with time.

Then, each trajectory sample has three kinds of wind :

1) Predicted Wind

2) True Wind

3) Updated Wind (in case of lack of neighbor, such

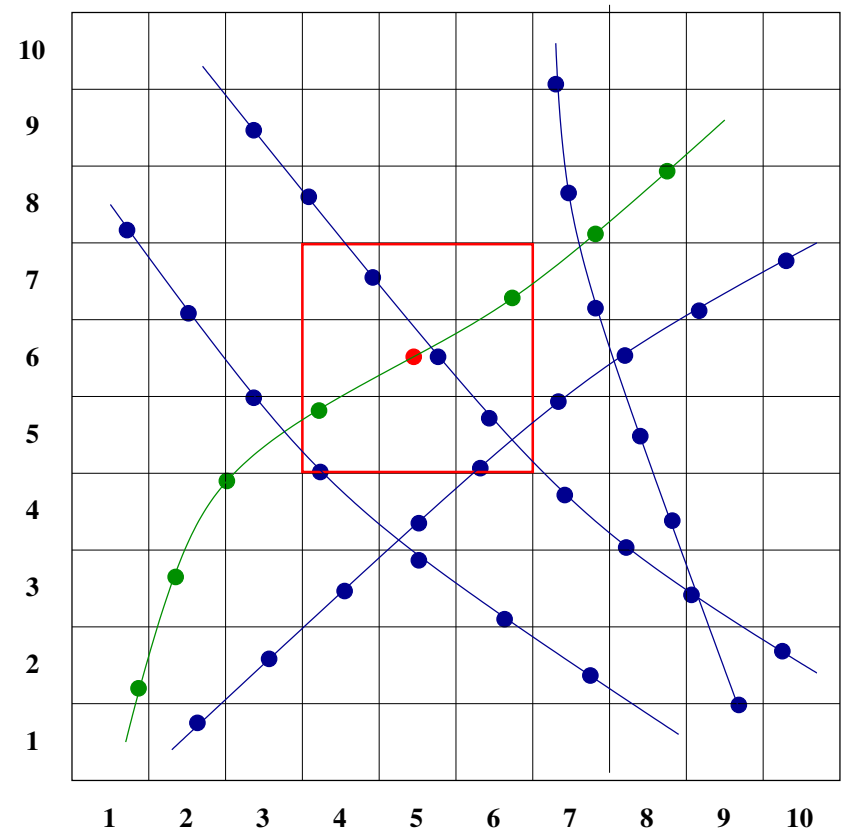

Figure 4. Grid used for neighbor detection

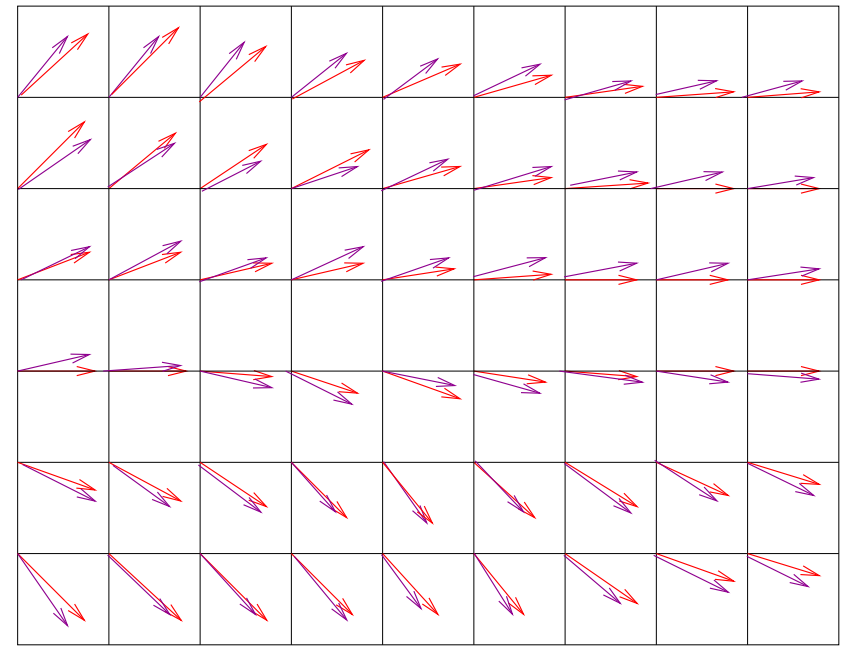

Figure 5. Predicted \& true wind grid

Updated Wind is equal to the Predicted Wind, meaning there is no improvement).

In order to improve the updated wind computation process, a wind interpolation algorithm has been included which interpolated the updated winds.

Having some wind estimates on some points in the airspace located in the neighborhood of an aircraft, the next step is to build a local wind field. In order to interpolate wind measures we propose to use a non linear dynamical system modeling.

We first consider measures from others aircraft 
(blue arrows as shown in Figure 6)

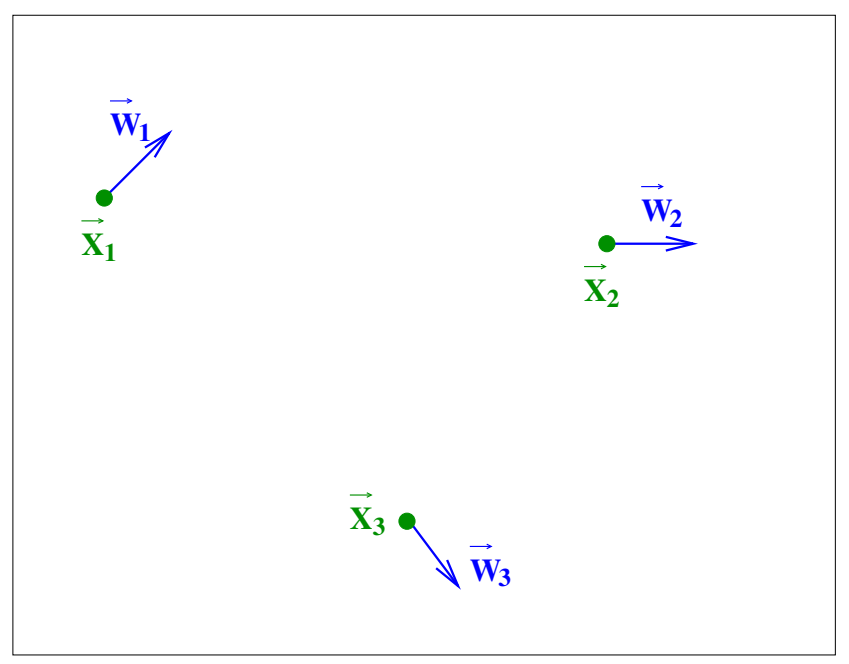

Figure 6. Other aircraft measures

Then, a grid is built where the wind field will be computed (Figure 7).

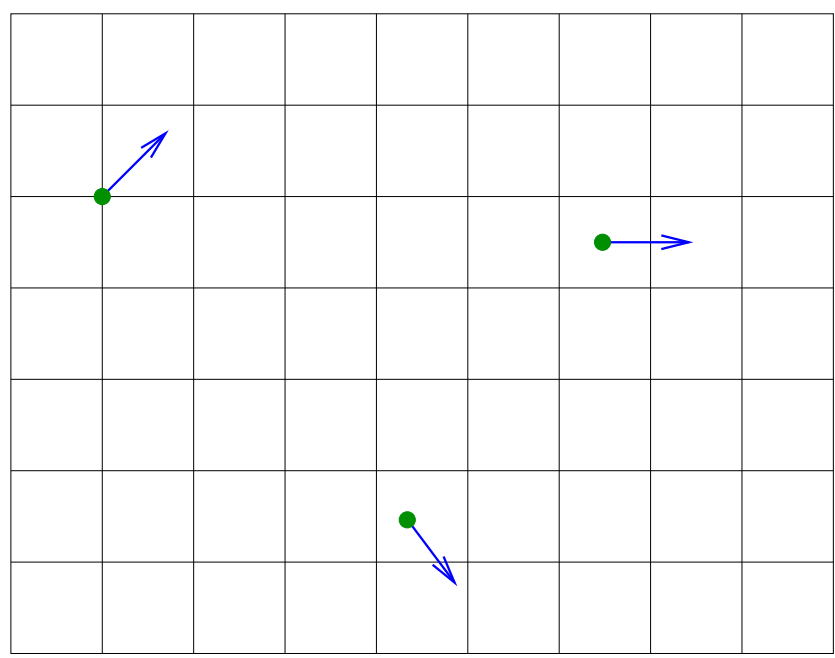

Figure 7. Wind field grid computation

On such grid, the wind field is then computed as shown on Figure 8.

To build such a wind field, a non linear dynamical system summarized by the following equation has been used :

$$
\dot{\vec{X}}(t)=\vec{f}(\vec{X})
$$

where $\vec{X}$ is the state vector of the system $(\vec{X}=$ $[x, y, z]^{T}$ ) and $\vec{f}: C^{2}$ the vectors field, describe systems which integral curves may fit the observed trajectories. This equation associates a vector speed $\dot{\vec{X}}$ to a position in the space coordinate $\vec{X}$ and then

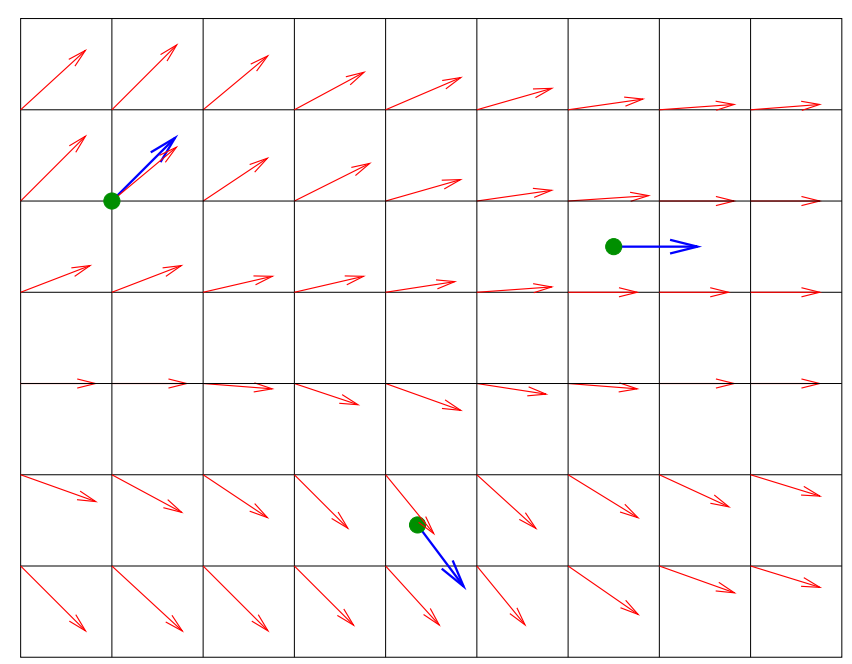

Figure 8. Wind field interpolation

synthesizes a particular vectors field. Based on the observations of the aircraft (positions, speed vectors), the dynamical system has to be adjusted with the minimum error. This fitting is done with a Least Square Minimization (LMS) method for which the following criterion is used :

$$
E_{1}=\sum_{i=1}^{i=N}\left\|\vec{V}_{i}-\vec{f}\left(\vec{X}_{i}\right)\right\|^{2}
$$

where $N$ is the number of observations.

Figure 9 flow chart summarizes our algorithm.

\section{Results}

In order to validate this concept we have considered a day of traffic over France for August 12, 2014. For this day, 8543 flights have been registered and we had the wind map predictions, thanks to Meteo France. We have considered the first map as the wind prediction time stamped $h$, and in order to simulate a real wind we have considered the second map time stamped $h+3$ hours as the true wind. An example of such wind map is given on figure 10 .

The 8000 flights have been simulated with such winds. Based on the associated flight plans, we first build the aircraft trajectories by using a fast time simulator based on Eurocontrol BADA data base. Such reference trajectories are simulated with the 


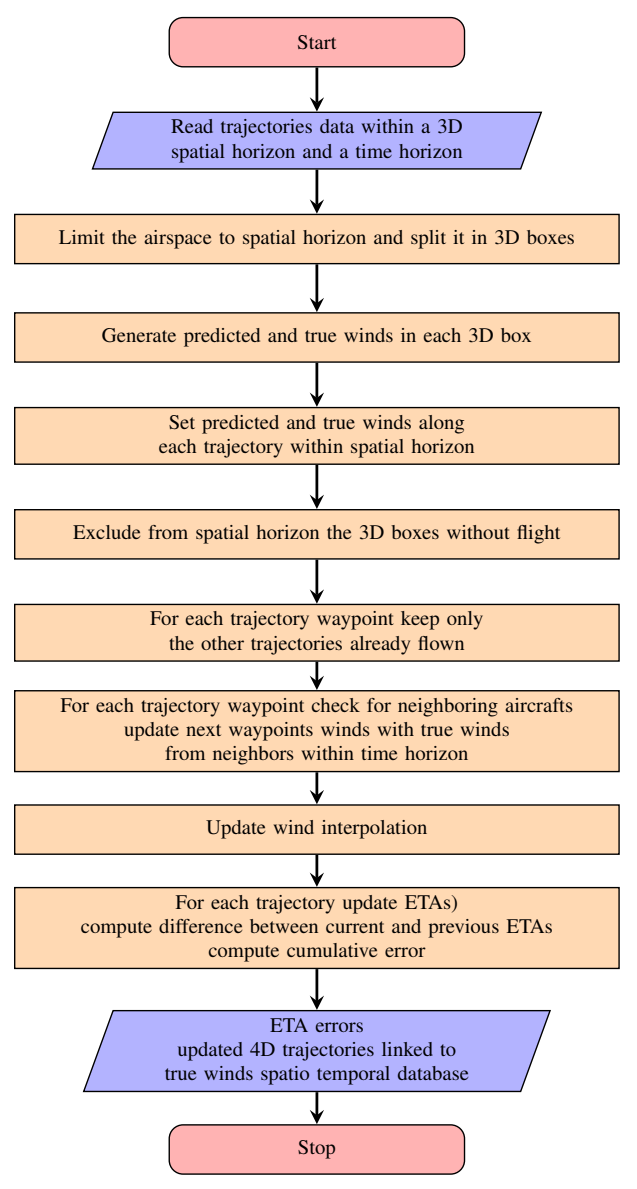

Figure 9. Algorithm block diagram

"true wind". For each trajectory, we compute the trajectory prediction by using the first wind map which corresponds to the "Pred-wind". Then, depending of the neighbor aircraft, the "updated wind" is also computed at each trajectory sample. Based on those three wind values, two performance analysis have been performed. The first one measures the benefit of the Wind Networking on the wind estimates along trajectories, the second one measures the associated benefits on the trajectory prediction performance.

\section{Wind Estimates Performances}

For each trajectory sample, three winds value have been stored (see figure 11) :

- True Wind

- Predicted Wind

- Updated Wind

Initially, the updated wind is set to the Predicted Wind and if an aircraft has neighbors, this wind is updated according to the winds measured by the

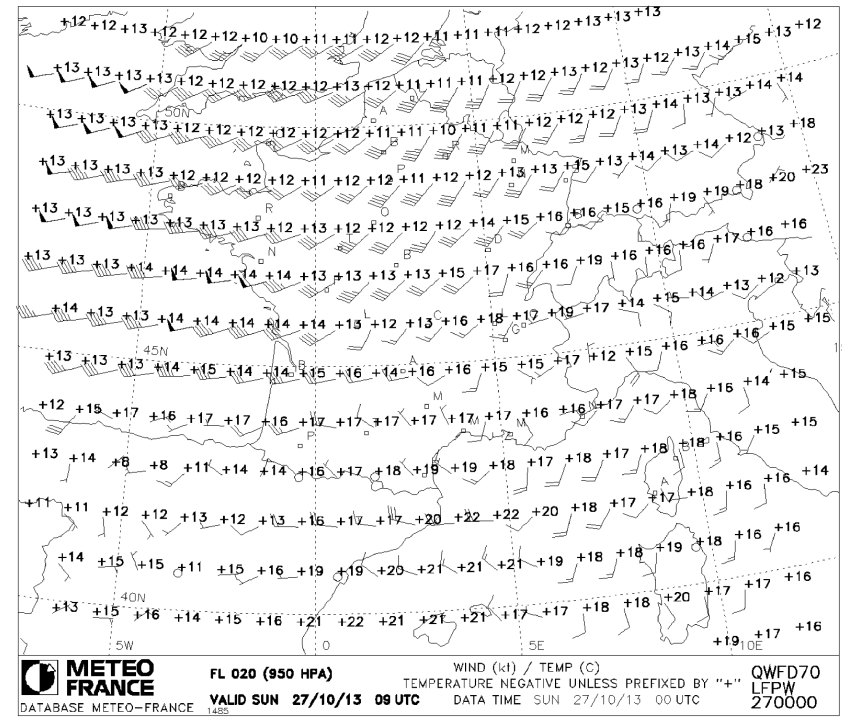

Figure 10. Example of wind map

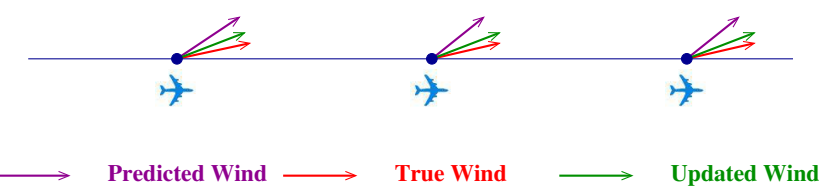

Figure 11. True, predicted \& updated winds

other aircraft. This updated wind will be used for the trajectory prediction.

Having those three winds along the trajectory, it is possible to compute wind errors. The error is linked to the predicted wind (we will consider the norm) :

$$
\text { PredWindError }=\| \text { PredWind }\|-\| \text { TrueWind } \|
$$

Having computed this error for each trajectory sample, it is possible to build a "WindPredError map" (see Figure 12) where the wind prediction error on each trajectory sample is represented. The former information is given in three dimensions but is here represented as a $2 \mathrm{D}$ graph. The error is computed in terms of norm. The red areas indicate an error of 15 knots. The red dots represent the areas with the biggest errors and the blue dots those with the smallest errors.

This computation has also been done for the UpdatedWindError :

UpdatedWindError $=\|$ U pdatedPred $\|-\|$ TrueWind $\|$

The associated map is given on Figure 13. We can notice that the red dots have disappeared in high traffic 


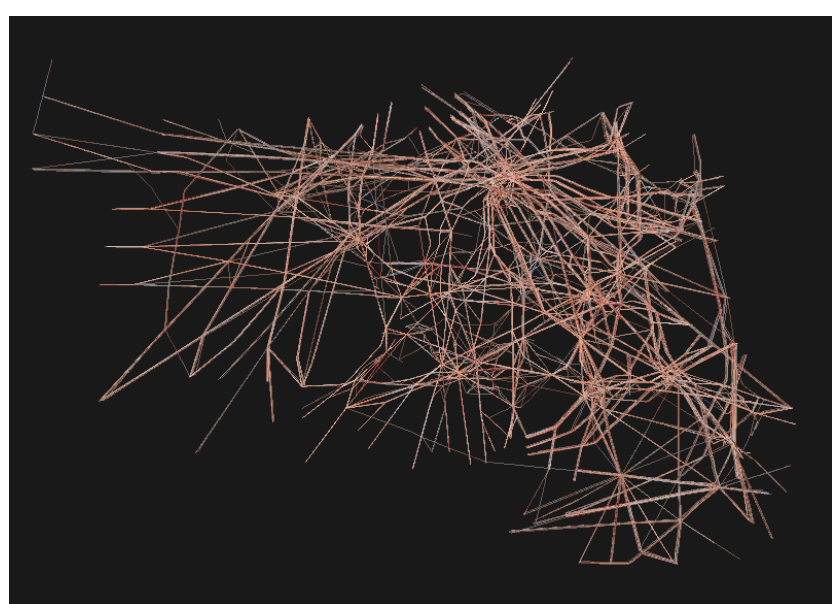

Figure 12. Per trajectory wind prediction error

density areas, and that we have much more blue areas, mainly in the high traffic density areas. The aircraft located in low traffic density areas do not benefit from other aircraft data and do not improve their wind estimates (but their needs for wind updating is less critical as the conflict risk is lower because the traffic spreads out).

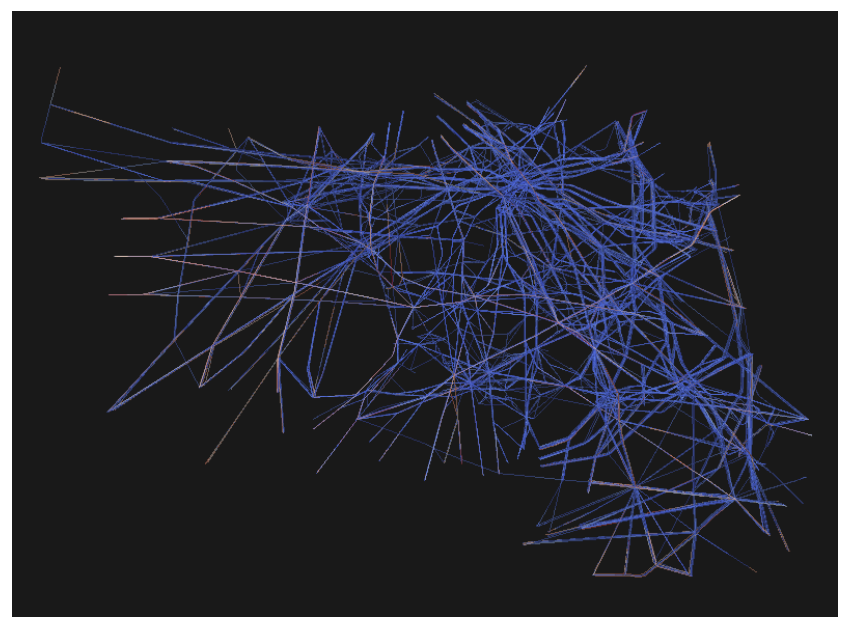

Figure 13. Per trajectory updated wind error

Finally, we have computed the Wind Networking Improvement by computing the difference between wind error:

Improvement $=$ PredWindError $-U$ pdatedWindError

This value is positive and is higher when the improvement is also higher. As for the previous values, we can also compute a map for this improvement (see Figure 14). We have just changed the color representation by setting green color for large improvement, thus the green areas locate where wind networking brings the most improvement (high traffic density areas).

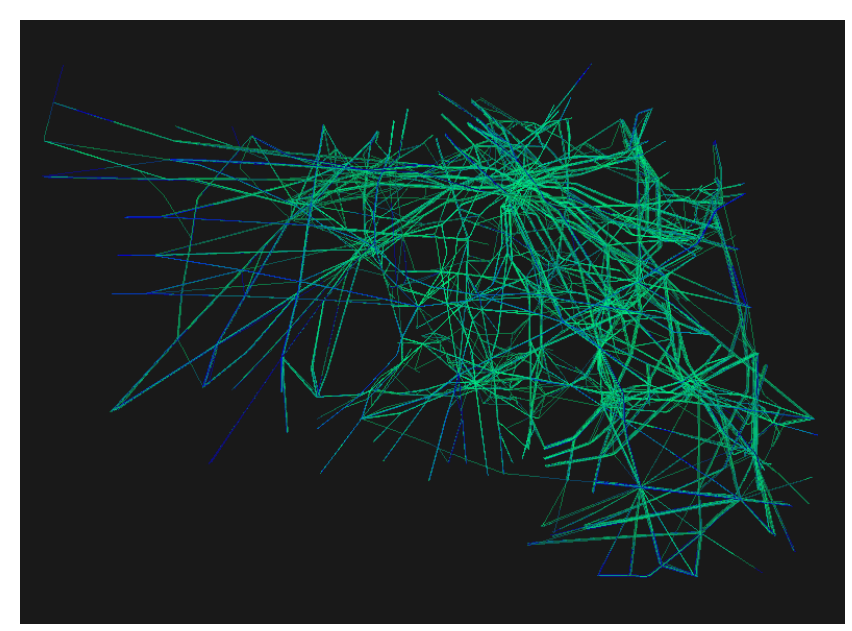

Figure 14. Wind estimate improvement areas

The second analysis we have performed is linked to the impact of the number of aircraft on the Wind Networking performances. For that we consider several aircraft densities and we compute the mean value of each error. The Table I summarizes those results.

Table I. Pred \& Updated WindError

\begin{tabular}{|c|c|c|c|c|c|}
\hline NbTraj & 100 & 1000 & 3000 & 5000 & 8000 \\
\hline PredErr (kts) & 5.11 & 5.13 & 5.12 & 5.11 & 5.14 \\
\hline UpdatedErr (kts) & 3.23 & 0.77 & 0.64 & 0.5 & 0.48 \\
\hline
\end{tabular}

For those experiments, we took the first 100 trajectories of the day, then the first 1000 and so on. With the first 1000 trajectories, the impact of the Wind Networking is already significant, the wind error drops down from $5.13 \mathrm{kts}$ to $0.77 \mathrm{kts}$.

\section{Trajectory Prediction Performances}

In order to validate the trajectory prediction performance, we consider that aircraft have to predict their future position at a given horizon all along their trajectory. As shown on Figure 15, at a given location, an aircraft predicts the time it will pass a given point on the future trajectory. Three times have been computed : the True Time, the Predicted Time and the Updated Time.

For a given location, three times are computed :

- True Time

- Predicted Time 


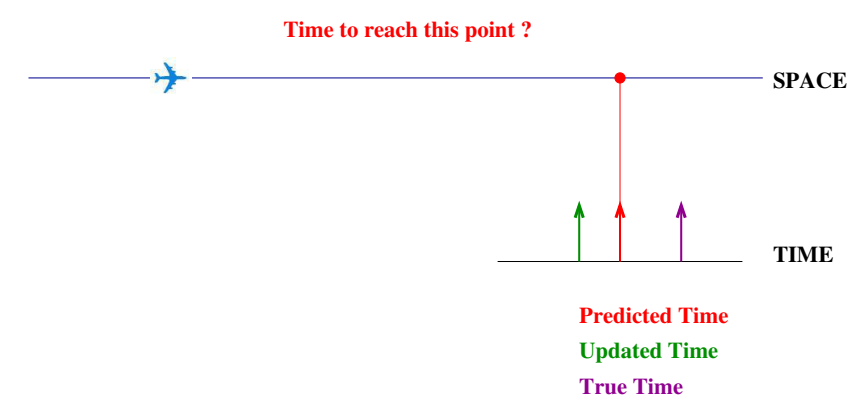

Figure 15. Aircraft position time estimates

- Updated Time

We compute also the following errors :

PredTimeError $=\mid$ PredTime - TrueTime $\mid$

U pdatedTimeError $=\mid$ U pdatedTime - TrueTime $\mid$

For different prediction horizon time (HT), we have computed the average Predicted Time Error and the associated Updated Time Error (see Table II).

\section{Table II. Predicted \& Updated Time Errors}

\begin{tabular}{|c|c|c|c|c|c|c|}
\hline HT & 5 & 10 & 15 & 20 & 30 & 45 \\
\hline PreDErr $(\mathrm{sec})$ & 4.5 & 9 & 13.3 & 16.8 & 20.3 & 22.4 \\
\hline UpdErr $(\mathrm{sec})$ & 0.4 & 0.8 & 1.3 & 1.8 & 2.2 & 2.7 \\
\hline
\end{tabular}

As we can see, the improvement on the Time Error is significant too.

\section{Conclusion}

Beyond operational concerns, flight safety as a main goal needs also accurate TP. Some accidents (Controlled Flight into Terrain (CFIT), collision, ...) or incidents (loss of separation, wake vortex encounter, airspace infringement, ...) were due to poor TP.

As planned in the future ATM concepts (SESAR and Nextgen), the concept of 4D Trajectory Based Operation will be the cornerstone of those new systems. In this 4D TBO framework, one must be able to locate accurately aircraft in the $4 \mathrm{D}(3 \mathrm{D}+\mathrm{T})$ space in order to improve traffic synchronization, sequencing and merging, overload detection, etc...

In order to reach these goals, trajectory prediction has to be improved so as to reduce the uncertainty of the future position of aircraft. One of the major Trajectory Prediction limiting factor is the wind along the future trajectory.
Aircraft at their current position, measure the wind with a very good accuracy and based on the future technology, it is reasonable to consider that aircraft would be able to share this wind information shortly with ground (e.g Maastricht Upper Area Control Centre Controller-Pilot Data Link Communication $(C P D L C))$ and other aircraft.

In this paper we have developed a Wind Networking concept in order to improve the trajectory prediction. In a first part, this concept has been described and we have investigated the potential applications for Air Traffic Management. We have proposed an algorithm to simulate this concept, in which we have also proposed a methodology for wind measures interpolation.

The concept has then been tested on a realistic airspace (France) with 8000 flights, including short, medium and long haul ones. The improvement on both wind estimate and trajectory prediction has been demonstrated with very hopeful results.

Future research will also measure the impact of the Wind Networking Concept on the conflict detection improvement. We will also investigate the possibility to improve the temperature estimate by the same kind of concept. As a matter of fact temperature also influences the Trajectory Prediction by the mean of air density, and may also be of some interest for carriers when choosing their optimal flight levels.

\section{References}

[1] C. SESAR, SESAR Master Plan, 2008.

[2] J. Planning and D. O. JPDO, Concept of Operations for the Next Generation Air Transportation System, 2006.

[3] CIVIL AVIATION BUREAU OF JAPAN, The Long-Term Vision of Future Air Traffic Systems in Japan - CARATS, 2010.

[4] OACI, 2013-2028 Global Air Navigation Plan Doc 9750-AN/963, ISBN 978-92-9249-365-3, 2013.

[5] Saulo Da Silva, Trajectory-Based Operations (TBO), ICAO Workshop on preparations for ANConf/12 - ASBU methodology, 2012.

[6] Stéphane MONDOLONI, Aircraft trajectory prediction errors, FAA/Eurocontrol Action Plan 16, Common Trajectory Prediction Capabilities, 2006. 
[7] S. Mondoloni and D. Liang, "Improving trajectory forecasting through adaptive filtering technique," in Proceedings of $5^{\text {th }}$ USA-Europe ATM Seminar, FAAEurocontrol, 2003.

[8] R. Cole and al., "Wind prediction accuracy for air traffic management decision support tools," in Proceedings of $3^{\text {th }}$ USA-Europe ATM Seminar, FAAEurocontrol, 2000.

[9] C. Rekkas, C. Lefas, and N. Krikelis, "Three dimensional tracking using on-board measurements.," IEEE Transactions on Aerospace and Electronic Systems, vol. 27, no. 4, pp. 617-624, 1991.

[10] D. Delahaye, "Wind field update using radar track data," Master's thesis, Ecole Nationale de l'Aviation Civile, 1992.

[11] Bradford, "Using aircraft radar tracks to estimate winds aloft.," The Lincoln Laboratory Journal, vol. 2, 1989.

[12] Rodionova, Olga, Delahaye, Daniel, Sbihi, Mohammed, and Mongeau, Marcel, "Aircraft trajectory prediction in North Atlantic Oceanic Airspace by Wind Networking," in DASC 2014, 33rd Digital Avionics Systems Conference, Best Paper of Session \& 2nd Place Best Graduate Student Paper, Colorado Springs, United States, Oct. 2014. [Online]. Avail- able: https : / / hal - enac . archives - ouvertes . fr / hal 00996689.

[13] M. Jackson, "Standards for air traffic data. communication services. an overview for e-operations workshop.," in RTCA Special Committee 214, 2007. [14] Laurel Stell, "Prediction of top of descent location for idle-thrust descents," in Ninth USA/Europe Air Traffic Management Research and Development Seminar (ATM2011), 2011.

[15] D. Li Jin Yi Cao, "Investigation of potential fuel savings due to continuous-descent approach," Journal of aircraft, vol. 50, no. 3, pp. 807-816, 2013.

[16] G. Slater, "Study on variations in vertical profile for cda descents," in 9th AIAA Aviation Technology, Integration, and Operations Conference (ATIO), 2009. [17] R. Sopjes and P.M.A. de Jong and C. Borst and M.M. van Paassen and M. Mulder, "Continuous descent approaches with variable flight-path angles under time constraints," in AIAA Guidance, Navigation, and Control Conference, 2011.

34th Digital Avionics Systems Conference

September 13-17, 2015 